\title{
Contribution of inflammation and impaired angiogenesis to the pathobiology of chronic thromboembolic pulmonary hypertension
}

\author{
Rozenn Quarck¹, Marijke Wynants ${ }^{1}$, Erik Verbeken², Bart Meyns ${ }^{3}$ and \\ Marion Delcroix ${ }^{4}$
}

Affiliations: ${ }^{1}$ Respiratory Division, Dept of Clinical and Experimental Medicine, University of Leuven, Leuven, Belgium. ${ }^{2}$ Translational Cell \& Tissue Research, University of Leuven, Leuven, Belgium. ${ }^{3}$ Cardiac Surgery Dept, University Hospitals Leuven, Leuven, Belgium. ${ }^{4}$ Respiratory Division, University Hospitals and Dept of Clinical and Experimental Medicine, University of Leuven, Leuven, Belgium.

Correspondence: Rozenn Quarck, Respiratory Division, Department of Clinical and Experimental Medicine, KU Leuven, Herestraat 49, B-3000 Leuven, Belgium. E-mail: rozenn.quarckamed.kuleuven.be

ABSTRACT Deficient angiogenesis and systemic inflammation could be involved in the pathophysiology of chronic thromboembolic pulmonary hypertension (CTEPH). We aimed to characterise the histopathology of pulmonary vascular lesions from $52 \mathrm{CTEPH}$ patients who underwent a pulmonary endarterectomy (PEA) and investigate a potential link between clinical, biological and morphometric parameters.

Collagen, elastin, fibrin, lipid, endothelial, smooth muscle and inflammatory cell content was investigated using immunohistochemistry. Qualitative changes were evaluated using severity scores. Circulating levels of inflammatory mediators were measured using ELISA.

Neointima, thrombotic, recanalised and atherosclerotic lesions were found. Accumulation of macrophages, T-lymphocytes and neutrophils was found mainly in atherosclerotic and thrombotic lesions. Angiogenesis was observed in all kinds of lesions; low-scored angiogenesis predicted adverse outcome, including persistent pulmonary hypertension post-PEA, start of medical therapy and poor survival. C-reactive protein (CRP), interleukin-10, monocyte chemotactic protein-1, macrophage inflammatory protein-1 $\alpha$ and matrix metalloproteinase (MMP)-9 were significantly elevated in CTEPH patients. Plasma CRP and MMP-9 levels correlated with neutrophil and macrophage accumulation, respectively.

Enhanced systemic inflammation parallels local inflammatory cell infiltration in major pulmonary arteries at advanced stages of CTEPH. Impaired neovascularisation is associated with poor survival, start of medical treatment and persistent pulmonary hypertension post-PEA. These findings suggest that inflammation and impaired angiogenesis could contribute to the progression of the disease.

@ERSpublications

Inflammation and deficient angiogenesis are involved in the pathogenesis of CTEPH http://ow.ly/LHIUa

For editorial comments see Eur Respir J 2015; 46: 303-306 [DOI: 10.1183/13993003.00962-2015]

This article has supplementary material available from erj.ersjournals.com

Received: Jan 132014 | Accepted after revision: April 02 2015 | First published online: June 252015

Conflict of interest: Disclosures can be found alongside the online version of this article at erj.ersjournals.com

Support statement: This work has been supported by a research grant from Pfizer (IIR WS232506) and by the Belgian Funds for Cardiac Surgery.

Copyright OERS 2015 


\section{Introduction}

Chronic thromboembolic pulmonary hypertension (CTEPH) is one of the main causes of precapillary pulmonary hypertension. CTEPH is characterised by the presence of unresolved thromboemboli associated with fibrous stenosis in the major pulmonary arteries, resulting in obstruction of major pulmonary arteries, increased pulmonary vascular resistance (PVR), pulmonary hypertension and progressive right heart failure. It may be caused by massive pulmonary embolism and local formation of thrombi. Whereas precapillary arteriopathy may occur in nonoccluded and occluded lung areas [1], the obliteration of major pulmonary arteries, removable by pulmonary endarterectomy (PEA) up to subsegmental level, is the major feature observed in CTEPH $[2,3]$.

Several authors have reported on the histomorphometry and cellular content of pulmonary vascular material collected from patients who underwent PEA. ThistLethwaite et al. [4] proposed a surgical classification based on a macroscopic observation of the presence of fresh thrombotic material and of fibrotic tissue either at a proximal or more distal level. Arbustini et al. [5] described fibrous plaques, including angioneogenesis, and core-rich atherosclerotic plaques, consisting of immunoreactive material including cholesterol clefts, macrophages, T-lymphocytes and calcification. BERNARD and YI [6] performed a clinicopathological study of 200 consecutive PEA samples which evidenced organised thrombi, inflammation, cholesterol clefts, calcification and increased cellularity. The cellular protagonists of major-vessel pulmonary remodelling in CTEPH patients remain poorly characterised.

In addition, there is evidence supporting the involvement of systemic inflammation in the pathogenesis of CTEPH, including elevated prevalence of inflammatory diseases in CTEPH patients [7], prevention of resolution of thrombi in rodents following bacterial infection [8] or splenectomy [9] and elevated tumour necrosis factor- $\alpha$ plasma levels in CTEPH [10]. Elevated PVR is correlated with increased expression of CC chemokine ligand (CCL)2 in plasma and large pulmonary arteries of CTEPH patients [11]. Circulating levels of various cytokines including interleukin (IL)-1 $\beta$, IL-4, IL-8 and IL-10 are increased in CTEPH patients [12]. Increased expression of intercellular adhesion molecule-1 and raised serum levels of its soluble form are found in various conditions associated with endothelial activation, such as pulmonary hypertension [13]. Increased expression of CCL3 has been reported in lung biopsies from patients with pulmonary arterial hypertension (PAH) [14]. Elevated circulating biomarkers of tissue remodelling, such as matrix metalloproteinases (MMPs) [15], and disordered angiogenesis, mainly characterised by impaired vascular endothelial growth factor (VEGF) expression [16] have also been observed in pulmonary hypertension. This inflammatory concept is further supported by our recent observations that CTEPH patients display elevated circulating C-reactive protein (CRP) levels, which decrease significantly after PEA [17], and that CRP may increase endothelium dysfunction and the proliferating capacities of endothelial and smooth muscle cells isolated from PEA material $[18,19]$.

More recently, a concept of deficient angiogenesis in CTEPH has been proposed [20], based on the presence of angiogenic factors such as VEGF or basic fibroblast growth factor (bFGF) in resolving venous thrombi [21], and on the enhancement of venous thrombus recanalisation and organisation caused by VEGF in a venous thrombosis rat model [22]. In addition, a deficiency in angiogenic gene expression has been observed in PEA material [23].

We hypothesised that inflammation and angiogenesis could be involved in the pathogenesis of CTEPH. The purpose of the current study was to evaluate the histomorphometry and cellular content of major-vessel lesions from CTEPH patients and to investigate a potential link with clinical and biological parameters. Therefore, we have analysed major-vessel lesions from CTEPH patients who underwent a PEA, by immunohistochemistry, in parallel with the analysis of the systemic inflammatory mediator and growth factor profile.

\section{Material and methods}

Pulmonary vascular material was obtained from 52 patients diagnosed with CTEPH, who underwent a PEA between April 1, 2004 and January 31, 2009 at the University Hospital Leuven (Belgium). The study protocol was approved by the institutional ethics committee of the University Hospital Leuven and all participants gave written informed consent.

Details of the material and methods are available in the online supplementary material.

\section{Results}

Characteristics of the study population

The demographics and clinical characteristics at diagnosis of the $52 \mathrm{CTEPH}$ patients from whom pulmonary vascular material was obtained are reported in table 1. $40 \mathrm{CTEPH}$ patients (77\%) had a history of acute venous thromboembolism (VTE). Among the 52 patients, 18 patients (34.6\%) had at least one of the established thrombotic risk factors including lupus anticoagulant/antiphospholipid antibodies (15.4\%), 
TABLE 1 Study population characteristics

\section{CTEPH patients}

Subjects $\mathrm{n}$
Age years
Female
BMI kg.m ${ }^{-2}$
Ever smoking
AHT
DMII
History of pulmonary embolism and/or DVT
Coagulopathy
6MWD m
NYHA functional class

$\begin{array}{cc}52 & 20 \\ 58(54-62) & 54(49-59) \\ 60 & 50 \\ 27(25-28) & 27(25-29) \\ 65 & 55 \\ 33 & 20 \\ 5.8 & 15 \\ 77 & \text { NA } \\ 71 & \text { NA } \\ 363 \pm 122 & \text { NA } \\ 2.7(2.6-2.9) & \text { NA }\end{array}$

Data are presented as mean $(95 \% \mathrm{CI}), \%$ or mean \pm SD, unless otherwise stated. CTEPH: chronic thromboembolic pulmonary hypertension; BMI: body mass index; AHT: arterial systemic hypertension; DMII: diabetes mellitus type II; DVT: deep venous thrombosis; 6MWD: 6-min walking distance; NYHA: New York Heart Association; NA: not applicable.

protein S and C deficiency (13.5\% and 1.9\%, respectively), activated protein C resistance, including factor $\mathrm{V}$ Leiden mutation, prothrombin gene mutation and antithrombin deficiency (5.8\%). In addition, factor VIII was elevated in $9.5 \%$ of the patients. Finally, 16 patients (31\%) had hyperhomocysteinaemia $\left(>15 \mu \mathrm{mol} \cdot \mathrm{L}^{-1}\right)$. After surgery, endarterectomised patients displayed a decrease in mean pulmonary artery pressure (mPAP) $(-14 \pm 13 \mathrm{mmHg} ; \mathrm{p}<0.0001)$ and in PVR $\left(-570 \pm 388 \mathrm{dyn} \cdot \mathrm{s} \cdot \mathrm{cm}^{-5} ; \mathrm{p}<0.0001\right)$ and an increase in cardiac index $\left(+0.91 \pm 0.93 \mathrm{~L} \cdot \mathrm{min}^{-1} \cdot \mathrm{m}^{-2} ; \mathrm{p}<0.0001\right)$ (table 2). 12 (23\%) patients had persistent pulmonary hypertension after PEA, defined by $\mathrm{mPAP} \geqslant 35 \mathrm{mmHg} 2-3$ days after surgery. 12 patients started medical treatment for PAH after PEA: seven with bosentan; four with epoprostenol and one with iloprost. Moreover, eight (15\%) patients had received PAH medical treatment before PEA: five received bosentan and three received epoprostenol; in three of them MPAP was normalised and PAH medical treatment stopped after 1 week (epoprostenol), 4 months (epoprostenol) and 4 months (bosentan).

During the observation period, 12 patients displaying persistent pulmonary hypertension after PEA died. Six in-hospital deaths were observed: right heart failure $(n=2)$, lung reperfusion oedema $(n=1)$, inflammatory reaction $(n=1)$, bleeding $(n=1)$ and undetermined reasons $(n=1)$. The six later deaths were attributed to sudden death $(n=2)$, myocardial infarction $(n=1)$, sepsis $(n=1)$, undetermined reasons 3 6 years after PEA $(n=1)$ and heroin overdose 6 months after surgery $(n=1)$. Among patients with normalised hemodynamics after PEA, no deaths were observed. The median "age of the lesions", calculated as the time lapse between the first acute episode of pulmonary embolism and the PEA was 18 months (range 4 months to 15 years). The median time between first detection of pulmonary hypertension and PEA was 15 months with a range of 1 month to 9 years. The median time between CTEPH diagnosis with right heart catheterisation and PEA was 2.4 months (range 0.7-10.5 months); this time interval also corresponds to the period between blood collection and surgery.

\section{Morphometric analysis of thromboembolic disease}

The 103 specimens were classified macroscopically (table 3) according to Jamieson classification [24]. Four different kinds of lesion were observed, including neointima formation, atherosclerotic lesions, thrombotic

\begin{tabular}{|c|c|c|c|c|}
\hline & Pre-PEA & Post-PEA & Change post-pre & p-value \\
\hline RAP mmHg & $6(5-8)$ & $8(9-11)$ & $+3 \pm 6$ & 0.0002 \\
\hline mPAP mmHg & $46(43-49)$ & $33(30-36)$ & $-14 \pm 13$ & $<0.0001$ \\
\hline PVR dyn $\cdot s \cdot \mathrm{cm}^{-5}$ & 795 (696-907) & $299(258-347)$ & $-570 \pm 388$ & $<0.0001$ \\
\hline CI L. $\min ^{-1} \cdot \mathrm{m}^{-2}$ & $2.0(1.9-2.1)$ & $3.0(2.8-3.1)$ & $0.91 \pm 0.93$ & $<0.0001$ \\
\hline
\end{tabular}

Data are presented as geometric mean $(95 \% \mathrm{Cl})$ or mean $\pm S D$, unless otherwise stated. Pre-PEA: at diagnosis; post-PEA: at day 2-3; RAP: right atrium pressure; mPAP: mean pulmonary arterial pressure; PVR: pulmonary vascular resistance; $\mathrm{Cl}$ : cardiac index. 
TABLE 3 Surgical classification of pulmonary vascular material according to THISTLETHWAITE et al. [4]

\begin{tabular}{lccc} 
& Type I & Type II & Type III \\
\hline Right specimen & 28 & 15 & 9 \\
Left specimen & 15 & 30 & 6
\end{tabular}

Data are presented as $\mathrm{n}$. Type I: fresh thrombus in the main and/or lobar pulmonary arteries; type II: intimal thickening and fibrosis proximal to the segmental arteries; type III: disease within distal segmental arteries only.

lesions and recanalised regions (fig. 1). Neointima is characterised by the presence of $\alpha$-smooth muscle actin (SMA)-positive cells (fig. 1a). Atherosclerotic lesions displayed macrophage-derived foam cells containing lipid droplets (fig. 1b). Thrombotic lesions either contained fresh thrombi or were more organised (fig. 1c). Fresh thrombus was characterised by the presence of fibrin, which disappears from organised thrombi (fig. 2a and b). Recanalised lesions were characterised by the presence of neovessels (fig. 1d).

Whereas neointima formation was observed in $94 \%$ of the patients, atherosclerotic lesions were more rarely found (31\% of the patients); thrombotic and recanalised lesions were present in $75 \%$ and $63 \%$ of the patients, respectively (fig. 3a). Interestingly, in patients bridged with PAH medical therapy prior to PEA, atherosclerotic lesions were more often observed (63\% versus $25 \%, \mathrm{p}=0.03$ ) and thrombotic lesions were more rarely found $(25 \%$ versus $70 \%, \mathrm{p}=0.01)$. The distribution of the different type of lesions according to Jamieson surgical classification (fig. 3b) shows that mural thrombus was dominant in type I material, whereas neointima was dominant in type II material. Recanalised lesions were more frequent in type II compared to type I material and atherosclerotic lesions were marginal in both material types. Regarding the two patients harbouring type III material, thrombotic, neointima, atherosclerotic and recanalised lesions were observed. The endothelium monolayer was absent or disrupted in $50 \%, 70 \%$ and $80 \%$ of the
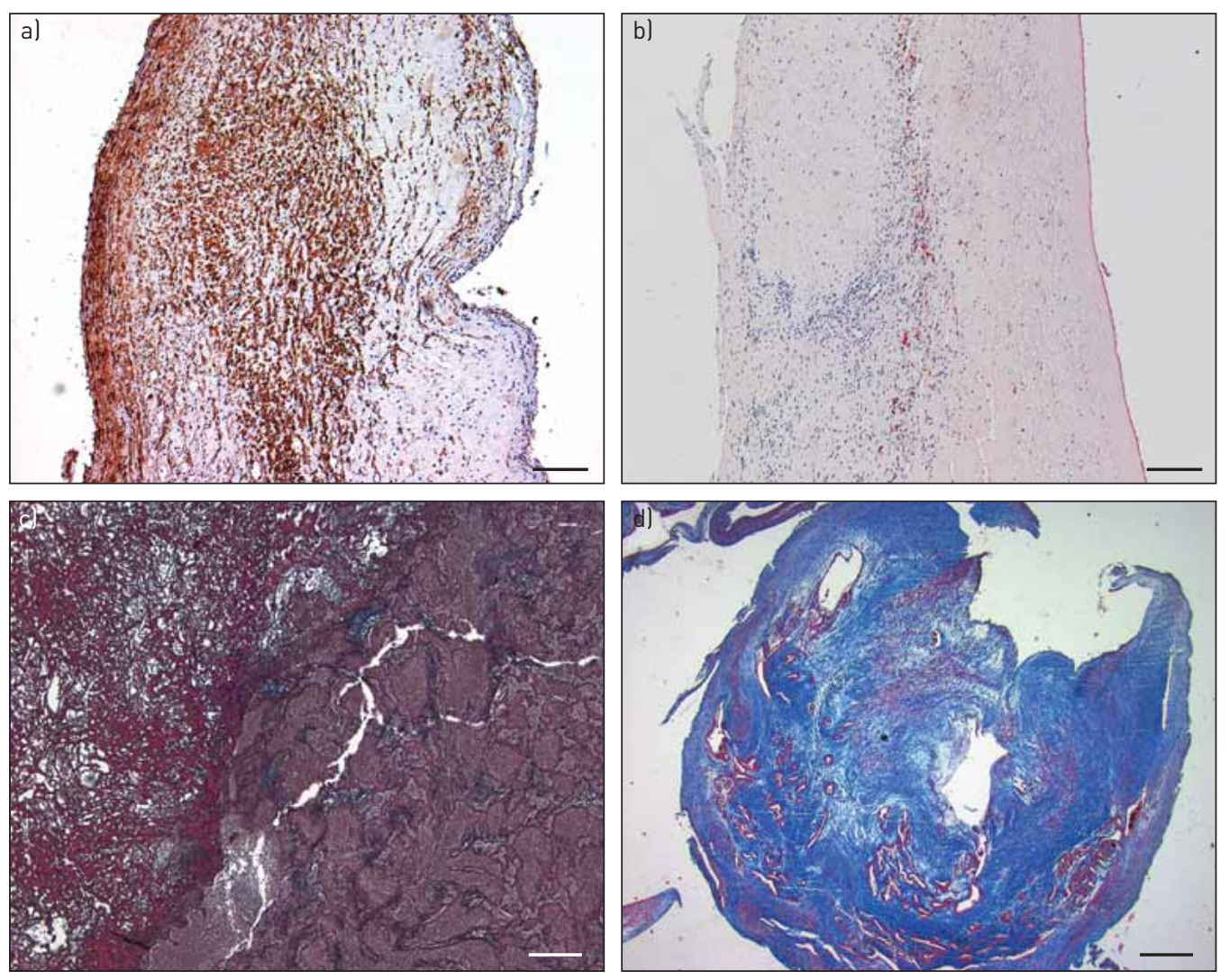

FIGURE 1 Different kinds of lesion observed in large pulmonary vascular material of patients with chronic thromboembolic pulmonary hypertension. a) Neointima immunolabelled by an anti- $\alpha$-smooth muscle actin antibody; b) atherosclerotic lesion labelled with oil red $\mathrm{O}$; c) thrombotic lesion labelled with phosphotungstic acid; d) recanalised lesion labelled using Masson staining. $\mathrm{a}-\mathrm{c}$ ) Scale bars $=400 \mu \mathrm{m}$; d) scale bar= $2 \mathrm{~mm}$. 

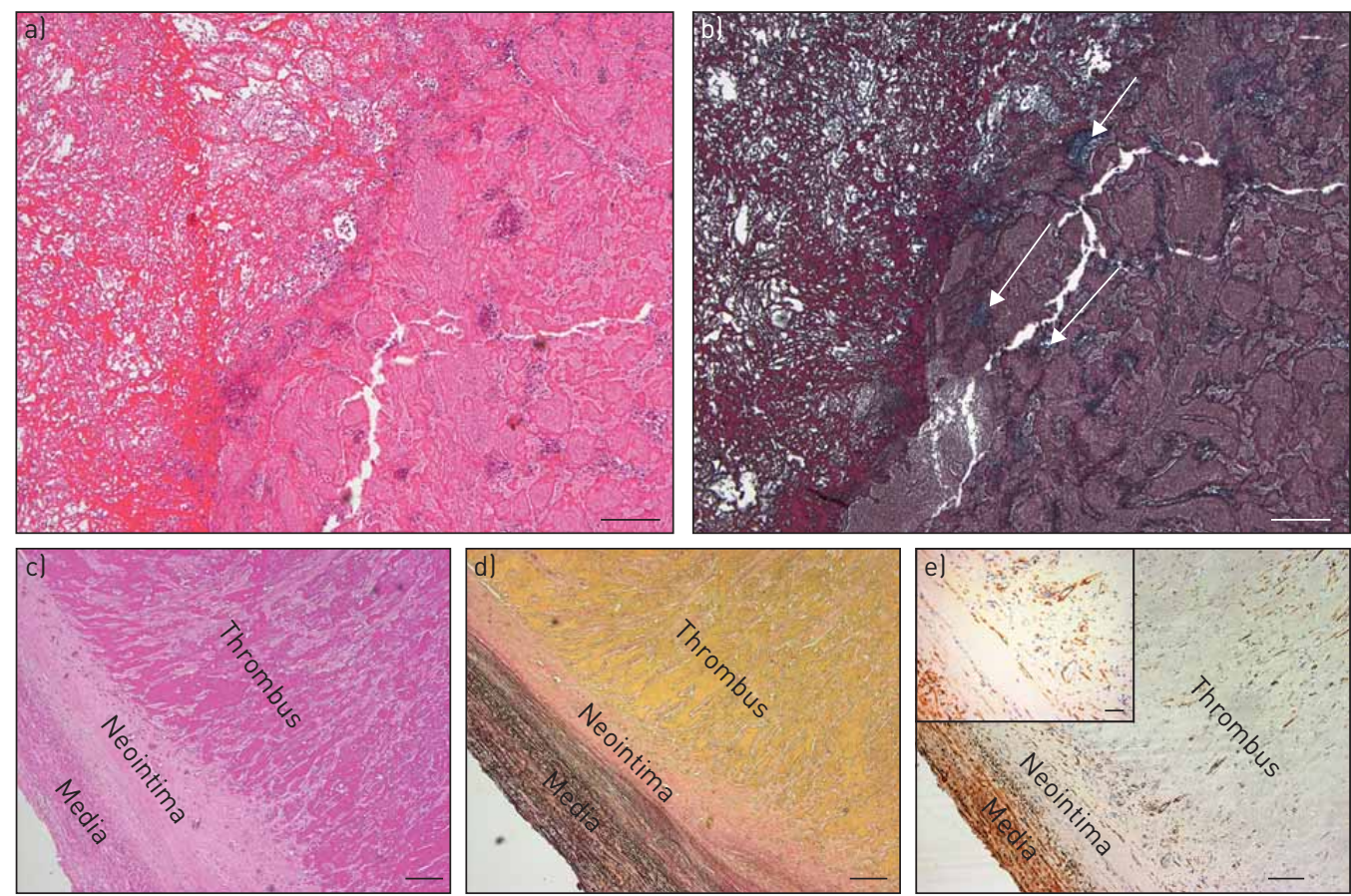

FIGURE 2 Histomorphometry of thrombotic lesions. a) Cell nuclei labelled with haematoxylin and eosin (HE); b) fibrin labelled with phosphotungstic acid (arrows); c) cells labelled with HE; d) elastin labelled using Verhoeff-van Gieson stain; e) smooth muscle cells immunolabelled using an anti- $\alpha$-smooth muscle actin antibody. a-e) Scale bars $=400 \mu \mathrm{m}$; e) inset scale bar $=100 \mu \mathrm{m}$.

neointima, atherosclerotic and thrombotic lesions, respectively. An intracellular lipid deposit was found in $90 \%$ of the analysed atherosclerotic lesions. Fresh thrombi were rarely observed in thrombotic lesions, whereas organised thrombi were found in $>90 \%$ of thrombotic lesions. Interestingly, colonisation of organised thrombotic lesions by intimal $\alpha$-SMA-positive cells was observed (fig. 2c-e). Among the 41 CTEPH patients with a documented history of VTE, we found the presence of mural thrombi in 26 (63\%) patients and no thrombi in 15 (37\%) of them. Similarly, among the 10 patients without history of VTE, six $(60 \%)$ had mural thrombi. Completeness of endarterectomy, evaluated by the number of thrombotic tails [25], was similar in patients with persistent pulmonary hypertension and in patients whose

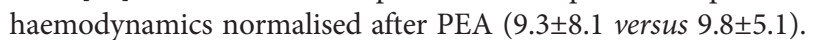

\section{Angiogenesis}

The presence of CD31-positive cells or endothelial cells was observed in all kinds of lesion. Angiogenic process was evidenced by the presence of isolated endothelial cells, sprouting capillaries (early stages) and developing and mature neovessels (late stages) (fig. 4). Only one patient displayed no angiogenesis and
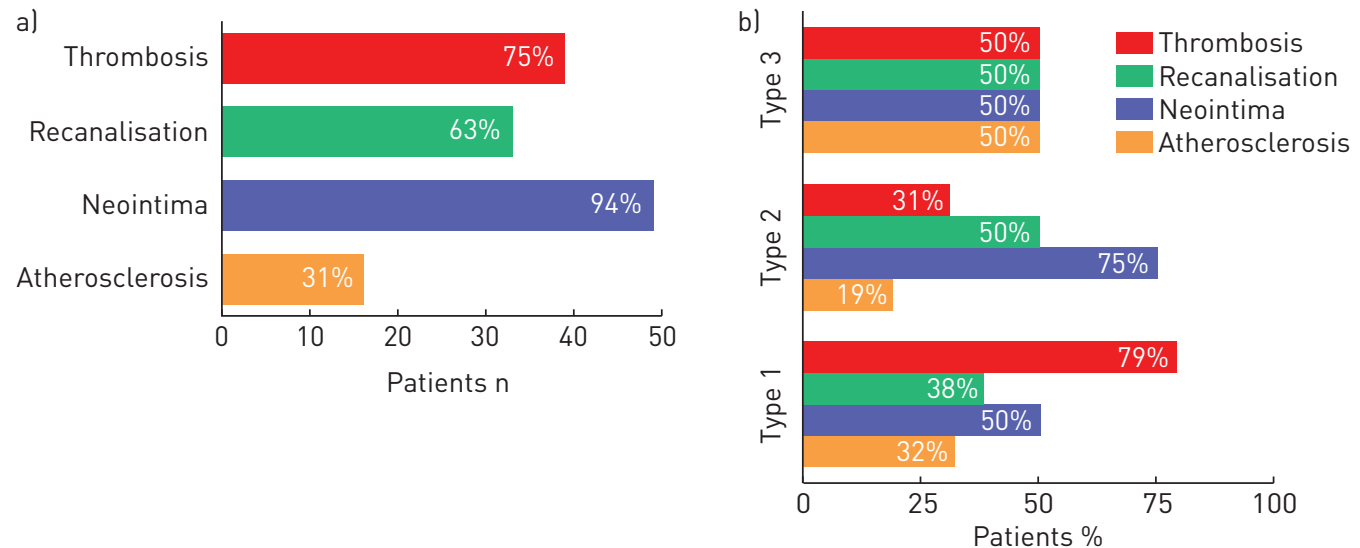

FIGURE 3 Repartition of the different kinds of lesion a) among patients and b) according to Jamieson surgical classification [4]. 

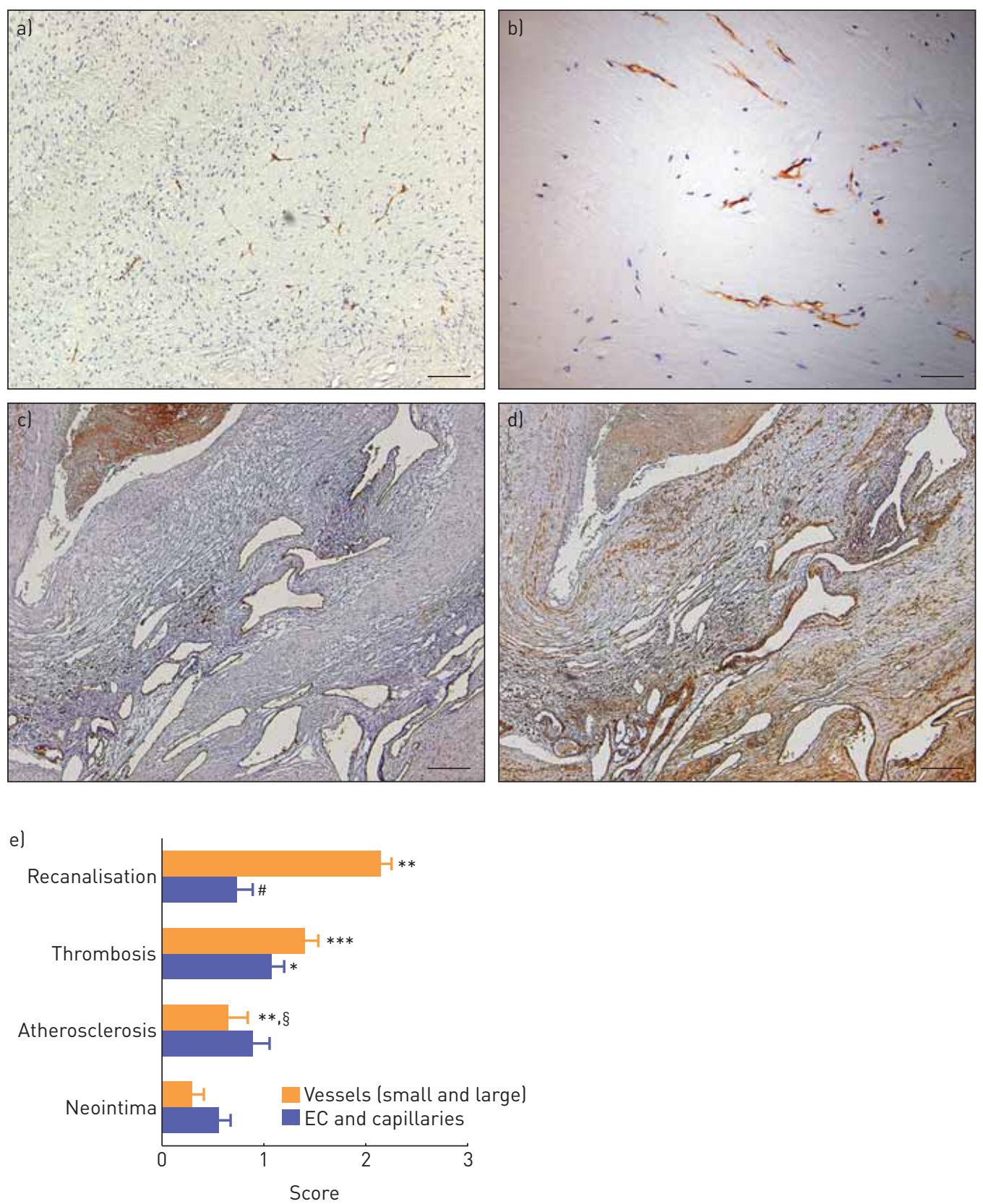

FIGURE 4 Angiogenesis. Different kinds of lesions were immunolabelled with an anti-CD31 antibody and a) cords, b) capillaries, c) small vessels and d) large vessels were identified. a, c, d) Scale bars=400 $\mu \mathrm{m}$; b) scale bar=200 $\mu \mathrm{m}$. e) CD31 immunolabelling was scored in the different kinds of lesion and for the different stages of angiogenesis. ANOVA for endothelial cells (EC) and capillaries: $\mathrm{p}=0.02 ;{ }^{*}: \mathrm{p}<0.05$ versus neointima; ${ }^{*}: \mathrm{p}<0.0001$ versus vessels. ANOVA for vessels: $\mathrm{p}<0.0001{ }^{* *}: \mathrm{p}<0.01$ versus thrombosis; ${ }^{* * *}: \mathrm{p}<0.001$ versus neointima; ${ }^{\S}: \mathrm{p}<0.001$ versus recanalisation.

four did not harbour late angiogenesis. Whereas the amount of isolated endothelial cells and capillaries was comparable in the four kinds of lesions, the number of neovessels progressively increased from neointima and atherosclerotic to thrombotic and recanalised lesions (fig. 4e). Moreover, in neointima and atherosclerotic lesions, cords and capillaries were predominant, whereas in thrombotic and recanalised lesions, neovessels were more represented, with a significant difference in recanalised lesions (fig. 4e). The overall angiogenesis score was significantly lower in nonsurviving CTEPH patients $(0.66 \pm 0.37$ versus $1.04 \pm 0.46, \mathrm{p}=0.009$ ) (online supplementary fig. $\mathrm{S} 1 \mathrm{~b}$ ), and tended to be lower in CTEPH patients with persistent pulmonary hypertension after PEA $(0.76 \pm 0.46$ versus $1.01 \pm 0.46, \mathrm{p}=0.10)$ and in CTEPH patients who started medical treatment $(0.80 \pm 0.44$ versus $1.03 \pm 0.46, \mathrm{p}=0.08)$, but did not reach statistical significance. More specifically, late angiogenesis was significantly lower in CTEPH patients with persistent pulmonary hypertension post-PEA, in the nonsurvivors and in those patients who started medical 
treatment $(0.65 \pm 0.64$ versus $1.20 \pm 0.78, \mathrm{p}=0.03 ; 0.49 \pm 0.44$ versus $1.26 \pm 0.80, \mathrm{p}=0.001$; and $0.73 \pm 0.67$ versus $1.11 \pm 0.45, \mathrm{p}=0.01$, respectively) (fig. $\mathrm{S} 1 \mathrm{~d}-\mathrm{f}$ ). By contrast, early angiogenesis was similar in all groups. In addition, Kaplan-Meier analysis showed that patients with an overall angiogenesis score $>0.85$ (fig. 5a) and with a late-scored angiogenesis $>0.80$ (fig. 5b) displayed a significantly higher rate of survival; patients with a late-scored angiogenesis $<0.70$ (fig. $5 \mathrm{c}$ ) harboured a greater risk of starting medical treatment. Finally, overall and late angiogenesis are both predictive for survival, start of medical treatment and persistent pulmonary hypertension post-PEA (fig. $5 \mathrm{~d}$ and e). Overall angiogenesis was inversely correlated with the age of the lesion $(\mathrm{r}=-0.57, \mathrm{p}=0.0001)$. Age of lesions was higher in nonsurvivors compared to that of survivors $(66 \pm 74$ versus $27 \pm 33$ months, $\mathrm{p}=0.04)$. Although patients who died in hospital harboured a lower angiogenesis score, analysis of odds ratios showed that low angiogenesis did not predict early in-hospital death. Scored angiogenesis was similar in patients bridged with PAH medical therapy prior to PEA and in patients who did not receive any PAH medical therapy prior to PEA.

$\alpha$-SMA-positive cells were found as isolated smooth muscle cells in the four types of lesion, and as organised neovessels only in recanalised lesions (fig. 6a). Isolated $\alpha$-SMA-positive cells were evenly distributed in neointima, atherosclerotic and thrombotic lesions, whereas significantly higher proportions were observed in recanalised lesions. Neovessels were mainly found in recanalised lesions and were almost absent from the other kinds of lesion (fig. 6b). Similar to angiogenesis, CTEPH patients with persistent pulmonary hypertension post-PEA displayed a significantly lower score for $\alpha$-SMA-positive cells ( $1.06 \pm 0.56$ versus $1.48 \pm 0.68, \mathrm{p}=0.03$ ). In addition, the amount of mature vessels was significantly lower in these patients $(0.23 \pm 0.50$ versus $0.84 \pm 0.85, \mathrm{p}=0.008)$, whereas the amount of isolated $\alpha$-SMA-positive cells was similar in the two subgroups of patients $(1.72 \pm 0.97$ versus $2.12 \pm 0.69, \mathrm{p}=0.095)$.

\section{Inflammatory cells}

B-lymphocytes, T-lymphocytes, macrophages and neutrophils were observed in the four kinds of lesion (fig. 7). Whereas B-lymphocyte accumulation was very limited, macrophages, neutrophils and T-lymphocytes were more abundant (fig. 8a). Atherosclerotic and thrombotic lesions displayed an elevated inflammatory cell accumulation compared to recanalised and neointima lesions (fig. 8b). Whereas B-lymphocytes, when present, were mainly rather deeply localised within the lesion and close to the native media, macrophages and T-lymphocytes were transmurally distributed and neutrophils were mainly found at the surface of the lesion, close to the residual lumen (fig. 8c). CTEPH patients with a history of VTE displayed a significantly higher score for CD68 (macrophages) compared to CTEPH patients without
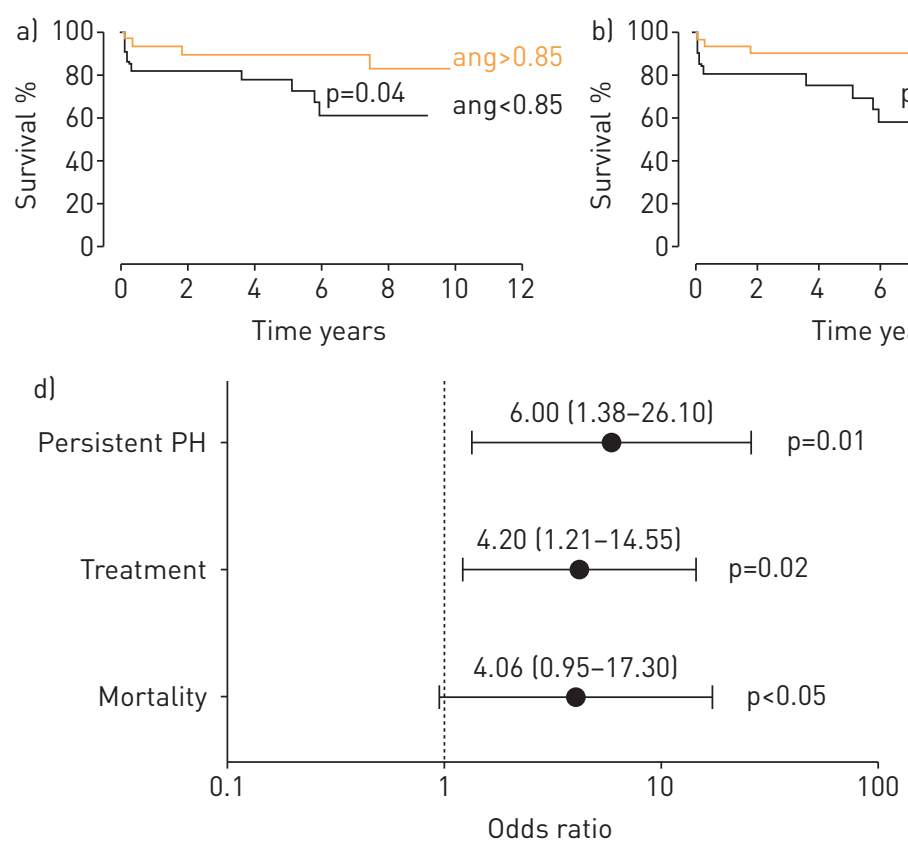
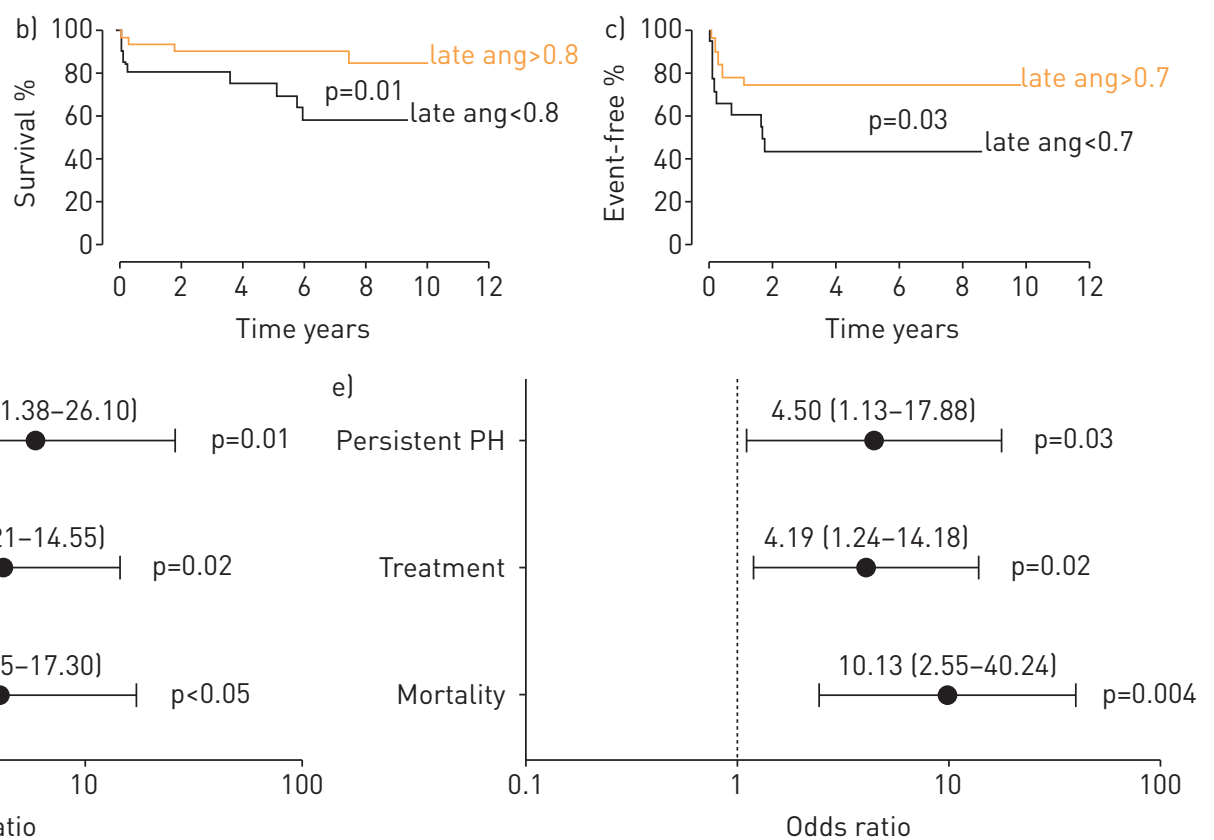

FIGURE 5 Angiogenesis and prediction of adverse outcome in chronic thromboembolic pulmonary hypertension. CD31 immunolabelling was scored in the

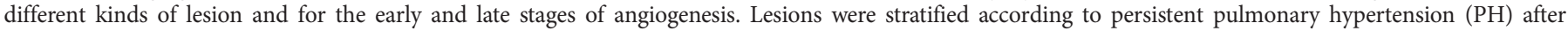

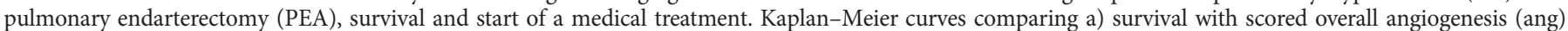
$<0.85$ or $>0.85$; b) survival with scored late angiogenesis $<0.80$ or $>0.80$; and c) start of a medical treatment with scored late angiogenesis $<0.70$ or $>0.70$ (cut-off values with best sensitivity and specificity upon receiver operating characteristic analysis). Odds ratios (95\% CI) of d) overall scored angiogenesis and e) late scored angiogenesis, evaluating the risk of persistent $\mathrm{PH}$ after PEA, start of a medical treatment and mortality. 

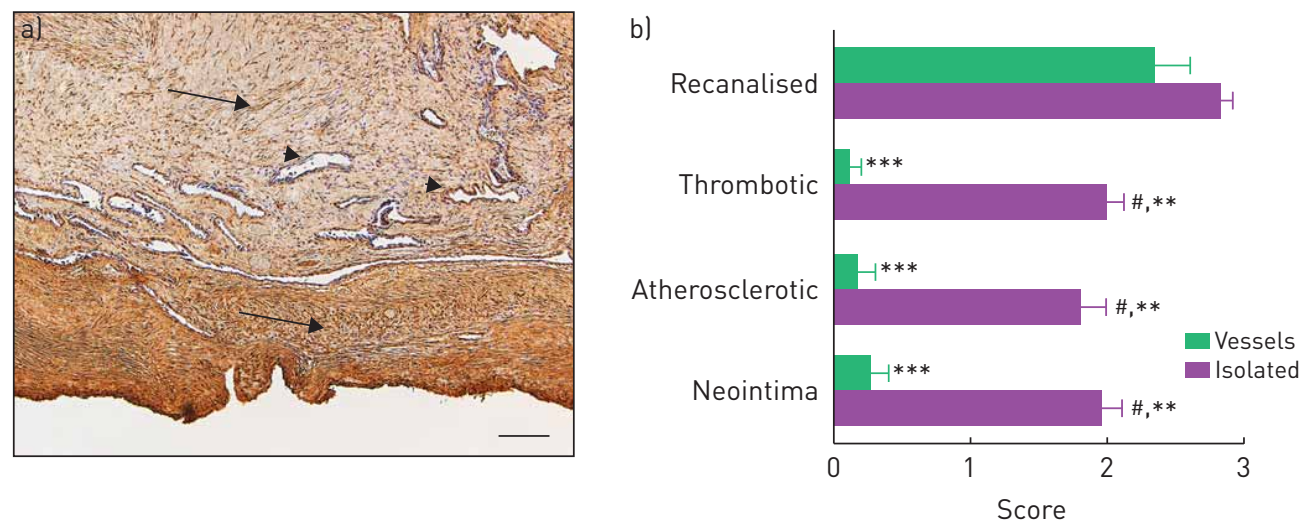

FIGURE $6 \alpha$-smooth muscle actin (SMA)-positive cell distribution. a) Lesions were immunolabelled with an anti- $\alpha$-SMA antibody and isolated smooth muscle cells (arrows) and smooth muscle cells organised in neovessels (arrowheads) were identified; scale bar $=400 \mu \mathrm{m}$. b) $\alpha$-SMA immunolabelling was scored into the different types of lesion and divided into isolated $\alpha$-SMA-positive cells and neovessels. ANOVA for neovessels $\mathrm{p}=0.003 ;{ }^{* * *}: \mathrm{p}<0.001$ versus recanalisation; ANOVA for isolated smooth muscle cells: $\mathrm{p}=0.0003$; ${ }^{* *}: \mathrm{p}<0.01$ versus recanalisation; ${ }^{*}: \mathrm{p}<0.0001$ versus neovessels.

history of VTE $(1.59 \pm 0.68$ versus $1.10 \pm 0.61, \mathrm{p}=0.04)$. In patients bridged with PAH medical therapy before PEA, T-lymphocytes scored accumulation was significantly lower $(0.88 \pm 0.27$ versus $1.66 \pm 0.13, \mathrm{p}=0.02)$.

\section{Circulating inflammatory and angiogenic mediators}

Plasma levels of inflammatory mediators measured in CTEPH patients and in healthy control subjects are presented in table 4. CRP, IL-10, monocyte chemotactic protein (MCP)-1, macrophage inflammatory protein (MIP)-1 $\alpha$ and MMP-9 are significantly higher in CTEPH patients compared to healthy control
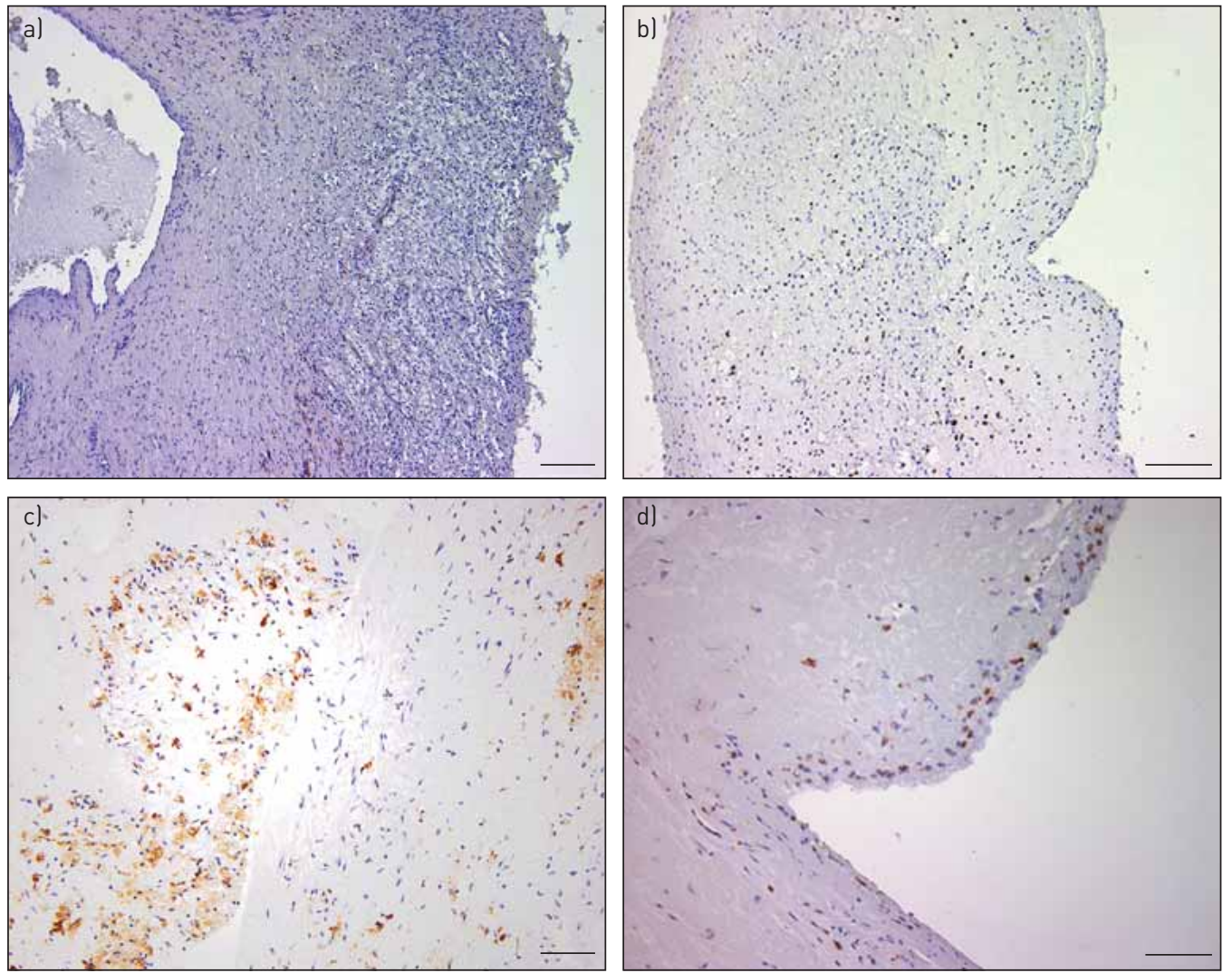

FIGURE 7 Inflammatory cells. Lesions were immunolabelled with a) anti-CD20 (B-lymphocytes); b) anti-CD3 (T-lymphocytes); c) anti-CD68 (macrophages); and d) anti-myeloperoxidase (neutrophils) antibodies. a, b) Scale bars $=400 \mu \mathrm{m} ; \mathrm{c}, \mathrm{d}$ ) scale bars $=200 \mu \mathrm{m}$. 

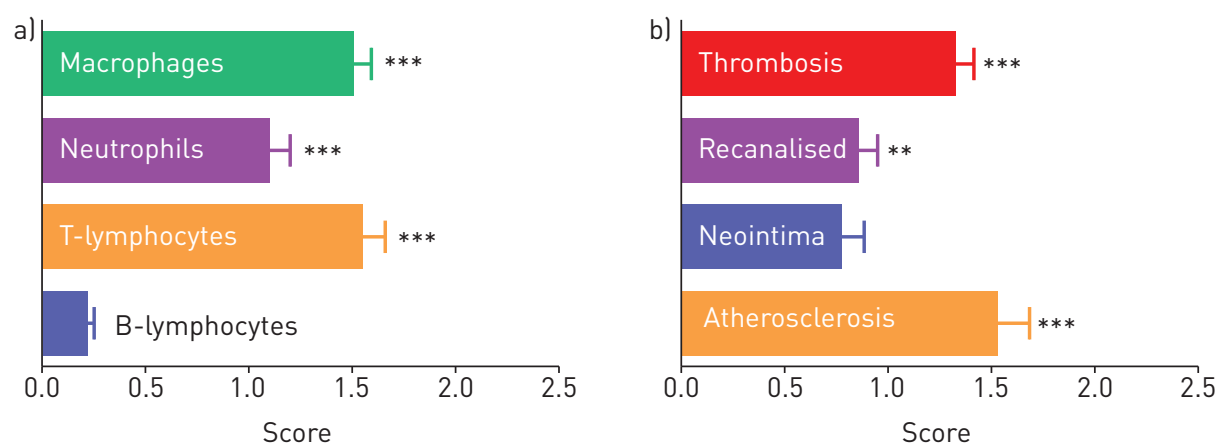

FIGURE 8 Inflammatory cell accumulation and topographic distribution. a) Inflammatory cell accumulation scores in different lesion types according to inflammatory cell type; ANOVA $\mathrm{p}<0.0001$; $^{* * *}$ : $\mathrm{p}<0.001$ versus B-lymphocytes. b) Inflammatory cell accumulation scores according to the type of lesion; ANOVA $\mathrm{p}<0.005 ;{ }^{* *}: \mathrm{p}<0.001$ versus neointima; ${ }^{* *}: \mathrm{p}<0.01$ versus atherosclerosis. c) Topographic distribution of inflammatory cells type; ANOVA $\mathrm{p}<0.0001$; ***: $\mathrm{p}<0.001$ versus $\mathrm{B}$-lymphocytes; ${ }^{*}: \mathrm{p}<0.001$ versus neutrophils.

subjects, whereas IL-1 $\beta$ and IL-6 levels were similar in the two groups. CRP and MMP-9 levels were positively correlated with inflammatory cell infiltration $(\mathrm{r}=0.34, \mathrm{p}=0.01$; and $\mathrm{r}=0.55, \mathrm{p}=0.01$, respectively) (online supplementary fig. S5A and C). More precisely, plasma CRP was correlated with neutrophil local accumulation ( $r=0.36, p=0.01$ ) (fig. S5B) and plasma MMP-9 was correlated with macrophage infiltration $(\mathrm{r}=0.54, \mathrm{p}=0.01$ ) (fig. S5D). VEGF levels tended to be lower in CTEPH patients without reaching statistical significance $\left(17.8,95 \%\right.$ CI $5.5-57.9$ versus 32.5 , 95\% CI 18.4-57.4 pg. $\mu \mathrm{L}^{-1} ; \mathrm{p}=0.09$ ). VEGF was significantly lower in CTEPH patients with persistent pulmonary hypertension post-PEA $(26.2 \pm 13.6$ versus 83.1 $\pm 17.0 \mathrm{pg} \cdot \mu \mathrm{L}^{-1}, \mathrm{p}=0.03$ ) as well as MMP-9 (5561 \pm 597 versus $\left.8566 \pm 1238 \mathrm{pg} \cdot \mu \mathrm{L}^{-1}, \mathrm{p}=0.04\right)$. Circulating VEGF and MMP-9 did not correlate with scored angiogenesis. Interestingly, VEGF was inversely correlated with mPAP measured 3 days after PEA $(\mathrm{r}=-0.51, \mathrm{p}=0.04)$ and VEGF $<48 \mathrm{pg} \cdot \mathrm{mL}^{-1}$ predicted persistent pulmonary hypertension post-PEA (OR 24.00, 95\% CI 1.74-331.00; $\mathrm{p}=0.0075$ ).

\section{Discussion}

In the present study, we analysed major-vessel lesions of CTEPH patients using immunohistochemistry and found the presence of four types of lesion: neointima, thrombotic, atherosclerotic and recanalised lesions. Accumulation of inflammatory cells, including T-lymphocytes, macrophages and neutrophils was found in all types of lesions. Inflammatory mediators including IL-10, MCP-1, MIP-1 $\alpha$ and MMP-9 were elevated in CTEPH patient plasma. An association between local and systemic inflammation was observed. Angiogenesis and $\alpha$-SMA-positive cells were observed in all types of lesions, with a predominance of neovessels in recanalised lesions. Interestingly, neovessels were less abundant in material from patients with postoperative persistent pulmonary hypertension, and low circulating levels of VEGF predicted

TABLE 4 Plasma levels of inflammatory proteins in chronic thromboembolic pulmonary hypertension (CTEPH) patients and healthy subjects

\begin{tabular}{|c|c|c|c|}
\hline & Healthy controls & СТEPH & p-value \\
\hline Subjects n & 20 & 20 & \\
\hline CRP $\mathrm{mg} \cdot \mathrm{L}^{-1}$ & $2.4(1.6-3.4)$ & $6.4(4.3-9.4)$ & 0.0005 \\
\hline $\mathrm{IL}-1 \beta \mathrm{pg} \cdot \mathrm{mL}^{-1}$ & $0.12(0.09-0.15)$ & $0.12(0.07-0.20)$ & 0.86 \\
\hline IL-6 pg.mL ${ }^{-1}$ & $0.66(0.43-1.02)$ & $1.17(0.63-2.20)$ & 0.11 \\
\hline $\mathrm{IL}-10 \mathrm{pg} \cdot \mathrm{mL}^{-1}$ & $0.25(0.17-0.37)$ & $0.71(0.37-1.32)$ & 0.006 \\
\hline $\mathrm{MCP}-1 \mathrm{pg} \cdot \mathrm{mL}^{-1}$ & $109(91-130)$ & $158(134-186)$ & 0.002 \\
\hline $\mathrm{MIP}-1 \alpha \mathrm{pg} \cdot \mathrm{mL}^{-1}$ & $8.7(7.3-10.5)$ & $14.2(11.6-17.2)$ & 0.0006 \\
\hline MMP-9 pg.mL ${ }^{-1}$ & $1.29(0.49-3.38)$ & $6.70(5.50-8.17)$ & 0.0009 \\
\hline VEGF-A $\mathrm{pg} \cdot \mathrm{mL}^{-1}$ & $32.5(18.4-57.4)$ & $17.8(5.5-57-9)$ & 0.09 \\
\hline
\end{tabular}

Data are presented as geometric mean $(95 \% \mathrm{CI})$, unless otherwise stated. Bold type represents statistical significance. CRP: C-reactive protein; IL: interleukin; MCP: monocyte chemotactic protein; MIP: macrophage inflammatory protein; MMP: matrix metalloproteinase; VEGF: vascular endothelial growth factor. 
postoperative persistent pulmonary hypertension, suggesting that impaired angiogenesis could predict postoperative persistent pulmonary hypertension.

As previously found $[5,6,26]$, we observed the presence of resident organised thrombi, fibroproliferative intima including atheroma and intimal inflammation in the pulmonary vascular material collected during PEA. The mechanisms leading to the remodelling of unresolved thromboemboli into fibrotic tissue, narrowing and obstruction of major pulmonary arteries, increased PVR and right ventricular dysfunction still remain to be unravelled.

\section{Thrombotic material}

Thrombotic risk factors are frequently observed in CTEPH patients [27] and ARBUstini et al. [5] reported recent thrombotic material in $86 \%$ of specimens. By contrast, we rarely observed fresh fibrinous clots, confirming the findings of BLAUwET et al. [26] and BERNARD and Yi [6], who retrieved fresh thrombi in no patients and in only $0.8 \%$ of the analysed specimens, respectively. These discrepancies could be attributed to subjective differences in the interpretation by the pathologist of the degree of organisation of each thrombotic specimen or to therapeutic anticoagulation, which is mandatory for $\geqslant 3$ months before PEA in our institution. Blauwet et al. [26] found evidence of the presence of thrombi in each resected specimen, whereas we observed thrombotic lesions in $75 \%$ of the patients and mural thrombi in $36 \%$ of the patients.

\section{Neointima and atherosclerosis}

Although intimal thickening was described as the result of thrombus reorganisation, accompanied by fibrotic tissue incorporation into the intimal layer [26], our findings indicated that neointimal lesions should be distinguished from evolutionary thrombotic lesions regarding angiogenesis and inflammatory cell content. Similarly, neointimal thickening should be distinguished from atherosclerotic lesions, considering their different inflammatory cell content. Atherosclerotic lesions are mainly characterised by accumulation of inflammatory cells, cholesterol clefts and collagen fibres [5,26], whereas the intimal layer mainly involves collagen and elastin fibre deposition, and $\alpha$-SMA-positive cells potentially deriving from dedifferentiated smooth muscle cells migrating from the media $[28,29]$ or from circulating progenitor cells $[30,31]$. Whereas a paucity of inflammatory cells was observed in the intimal layer, atherosclerotic lesions were massively infiltrated by macrophages, and to a lesser extent by neutrophils and T-lymphocytes.

\section{Systemic and local inflammation}

Infiltration of various inflammatory cells including macrophages, B- and T-lymphocytes and neutrophils was observed, as previously found $[5,6,26]$. By scoring inflammatory cell accumulation, we could quantify the inflammatory process within major-vessel lesions of CTEPH patients. Macrophages, T-lymphocytes and neutrophils were found in the different types of lesion, whereas B-lymphocytes were rarely found, except in thrombotic lesions. Whereas inflammatory cell infiltrates were often observed in atherosclerotic and thrombotic lesions, recanalised and intimal lesions were almost devoid of inflammatory cells, suggesting differential distribution of chemokines and cytokines within the lesions. Moreover, the topographic analysis revealed a superficial localisation, close to the residual lumen, of neutrophils, and a transmural distribution of macrophages and T-lymphocytes, whereas B-lymphocytes were mostly localised deep within the lesion, close to internal elastic lamina and native media. Since material collected during PEA refers to advanced lesions and consequently chronic inflammation, the presence of neutrophils, key players of the innate immune system, rapidly recruited at the injury site following a trauma and mostly considered as a hallmark of acute inflammation, was quite unexpected. The presence of macrophages and T-lymphocytes, also observed in plexiform lesions in $\mathrm{PAH}$ patients [32, 33], was predictable and illustrate a chronic inflammatory process. Accordingly, $\mathrm{CD}^{+}{ }^{+} \mathrm{T}$-lymphocytes were observed in CTEPH patients [34]. Interestingly, circulating inflammatory mediators, namely CRP, IL10, MCP-1, MIP-1 $\alpha$ and MMP-9 were elevated in CTEPH patients, suggesting mild chronic inflammation, as previously mentioned $[11,17]$.

In addition, among those enhanced inflammatory mediators, CRP and MMP-9 were found associated with local accumulation of inflammatory cells, namely neutrophils and macrophages, respectively. Bacterial infection, as well as splenectomy, by potentiating the inflammatory insult, could also contribute to progression of the disease [8,35]. Whereas KIMURA et al. [11] observed a correlation between circulating MCP-1 and PVR, we did not find any association between haemodynamic parameters and circulating inflammatory markers, nor with local inflammatory cell accumulation. By contrast, circulating CRP was correlated with local neutrophil accumulation. A correlation between plasma CRP and blood toxic granulation neutrophils has been established in inflammatory diseases [36, 37]. CRP has been shown to elicit white blood cell activation [38]. CRP could prevent neutrophil chemotaxis [39, 40], which could partially explain the neutrophil distribution at the surface of the lesion. Local production of CRP has been observed in vulnerable plaques and in injured coronary arterial walls [41] and in the major large pulmonary arterial tissue from CTEPH patients [18]. CRP-induced production of MMP-9 has been found 
in macrophages [42]. MMP-9 is produced by neutrophils, but can also activate neutrophils, and macrophage-derived MMP-9 may contribute to neovascularisation of ischaemic tissue [43].

\section{Angiogenesis and recanalisation}

In $>60 \%$ of the analysed lesions, we observed recanalised lesions characterised by fibrotic material including smooth muscle cells, collagen and elastin, and vascular neoangiogenesis, as previously described [5, 6]. One major feature of recanalised lesions is the presence of neovessels characterised by CD31-positive endothelial cells and $\alpha$-SMA-positive smooth muscle cells. Recanalisation is a process commonly involved in thrombus resolution and involves recruitment of various cell types including neutrophils, monocytes, endothelial cells and myofibroblasts [44]. For instance, this is illustrated by the colonisation of organised thrombi by migrating $\alpha$-SMA-positive cells (fig. 2e). It has been suggested that angiogenesis plays a key role in the resolution of thrombi in a rat model of venous thrombosis, highlighting a potential role of angiogenic factors such as VEGF and bFGF [21, 22]. Angiogenesis was observed in all analysed specimens, as previously observed [5], although neovessels were mainly present in thrombotic and recanalised lesions. Interestingly, we observed that patients with adverse outcome displayed fewer neovessels; we also found that low angiogenesis scores could predict adverse outcomes. In addition, plasma VEGF 1) tended to be lower in CTEPH patients compared to healthy subjects; 2) was lower in CTEPH patients with persistent pulmonary hypertension post-PEA; 3) was inversely correlated with mPAP measured 3 days after PEA; and 4) low VEGF predicted persistent pulmonary hypertension post-PEA. With respect to these findings, we may speculate that enhanced pulmonary arterial angiogenesis could be responsible for partial permeability of the obstructive material with maintenance of a preserved postobstructive vascular bed through/by circulating vasoactive factors. Alternatively, in patients displaying less angiogenesis in the removed surgical material, inaccessible distal fibrothrombotic material could be more abundant and less recanalised, resulting in persistent pulmonary hypertension post-PEA. Moser et al. [1] suggested that endothelial cells or platelets stimulated by the pulmonary hypertensive state could contribute to distal vessel remodelling by producing and releasing proliferative and/or anti-apoptotic mediators. Accordingly, LANG et al. [20] observed a deficiency of vessels in areas distal to occluded vessels and defective angiogenesis was shown to delay thrombus resolution [23]. Additionally, angiostatic factors, which cause changes in calcium homeostasis, consequently affecting pulmonary endothelial cell proliferation and migration, and vessel formation, could be involved in impaired calcium homeostasis and endothelial dysfunction in CTEPH [45]. Finally, impaired neovascularisation was associated with adverse outcome, including poor survival, start of medical treatment and persistent pulmonary hypertension post-PEA in CTEPH patients. Pulmonary hypertension-related mortality, such as right heart failure or reperfusion injury represented only half the inpatient deaths, and none of the later deaths were attributed to right heart failure. However, persistent pulmonary hypertension can complicate the course of intercurrent diseases such as inflammatory reaction, bleeding, myocardial infarction, or sepsis; consequently, all-cause mortality was selected [46].

\section{Limitations}

The authors are aware of the limited volume of surgeries, high incidence of mortality (11\%) and of postoperative pulmonary hypertension reported in this cohort $(23 \%)$. However, the current article reports on long-term outcome of patients operated upone 5-10 years ago. Results of surgery, with regard to mortality and persistent pulmonary hypertension, were then less favourable than currently, even in larger centres [47]. Experienced surgeons can currently remove more distal material, which could possibly influence the results of this study. However, whereas CTEPH with a poor outcome had an impaired angiogenesis in the major pulmonary arteries, no difference was observed in the number of thrombotic tails, suggesting that the way in which the surgical specimen was distally removed was not a major concern. Finally, it would have been of great interest to investigate the role of bronchial artery collaterals, since Hoey et al. [48] suggested that collateral vessels from the systemic circulation can grow to reperfuse areas distal to complete obstruction. However, it does not seem to be easy to distinguish bronchial artery collaterals, as recently evoked by DORFMÜLLER et al. [49], who developed an elegant technique using different coloured ink to discriminate systemic arteries, and which also requires the disposal of the complete lung, which is absolutely not the case with material withdrawn during PEA.

\section{Conclusion}

Although the present study is basically a descriptive pathological study, the scoring of the different lesions represents an alternative approach compared to previous published investigations. Moreover, elevated inflammatory mediators as well as the association between local and systemic inflammation at advanced stages of the disease highlight the potential role of chronic inflammation in the pathogenesis of CTEPH. Finally, angiogenesis as a potential predictor of postoperative persistent pulmonary hypertension is an interesting finding, which deserves further investigation. 


\section{Acknowledgements}

Marion Delcroix is holder of the Actelion chair for pulmonary hypertension at the University of Leuven (Leuven, Belgium). The authors thank Frederick Guns and Petra Janssens (University Hospitals, Leuven), and Alicja Ronisz (KU Leuven) for excellent logistical and technical support. The authors are also grateful to Leanda Vengethasamy (KU Leuven) for helpful contribution in revising the manuscript.

\section{References}

1 Moser KM, Bloor CM. Pulmonary vascular lesions occurring in patients with chronic major vessel thromboembolic pulmonary hypertension. Chest 1993; 103: 685-692.

2 McNeil K, Dunning J. Chronic thromboembolic pulmonary hypertension (CTEPH). Heart 2007; 93: 1152-1158.

3 Hoeper MM, Mayer E, Simonneau G, et al. Chronic thromboembolic pulmonary hypertension. Circulation 2006; 113: 2011-2020.

4 Thistlethwaite PA, Mo M, Madani MM, et al. Operative classification of thromboembolic disease determines outcome after pulmonary endarterectomy. J Thorac Cardiovasc Surg 2002; 124: 1203-1211.

5 Arbustini E, Morbini P, D'Armini AM, et al. Plaque composition in plexogenic and thromboembolic pulmonary hypertension: the critical role of thrombotic material in pultaceous core formation. Heart 2002; 88: 177-182.

6 Bernard J, Yi ES. Pulmonary thromboendarterectomy: a clinicopathologic study of 200 consecutive pulmonary thromboendarterectomy cases in one institution. Hum Pathol 2007; 38: 871-877.

7 Bonderman D, Jakowitsch J, Adlbrecht C, et al. Medical conditions increasing the risk of chronic thromboembolic pulmonary hypertension. Thromb Haemost 2005; 93: 512-516.

8 Bonderman D, Jakowitsch J, Redwan B, et al. Role for Staphylococci in misguided thrombus resolution of chronic thromboembolic pulmonary hypertension. Arterioscler Thromb Vasc Biol 2008; 28: 678-684.

9 Frey MK, Alias S, Winter MP, et al. Splenectomy is modifying the vascular remodeling of thrombosis. J Am Heart Assoc 2014; 3: e000772.

10 Langer F, Schramm R, Bauer M, et al. Cytokine response to pulmonary thromboendarterectomy. Chest 2004; 126: $135-141$.

11 Kimura H, Okada O, Tanabe N, et al. Plasma monocyte chemoattractant protein-1 and pulmonary vascular resistance in chronic thromboembolic pulmonary hypertension. Am J Respir Crit Care Med 2001; 164: 319-324.

12 Soon E, Holmes A, Treacy CM, et al. Inflammatory cytokines are elevated in patients with operable chronic thromboembolic pulmonary hypertension and predict outcome post-endarterectomy. Am J Respir Crit Care Med 2011; 183: A2287.

13 Sungprem K, Khongphatthanayothin A, Kiettisanpipop P, et al. Serum level of soluble intercellular adhesion molecule- 1 correlates with pulmonary arterial pressure in children with congenital heart disease. Pediatr Cardiol 2009; 30: 472-476.

14 Fartoukh M, Emilie D, Le Gall C, et al. Chemokine macrophage inflammatory protein- $1 \alpha$ mRNA expression in lung biopsy specimens of primary pulmonary hypertension. Chest 1998; 114: 50S-51S.

15 Schumann C, Lepper PM, Frank $\mathrm{H}$, et al. Circulating biomarkers of tissue remodelling in pulmonary hypertension. Biomarkers 2010; 15: 523-532.

16 Tuder RM, Chacon M, Alger L, et al. Expression of angiogenesis-related molecules in plexiform lesions in severe pulmonary hypertension: evidence for a process of disordered angiogenesis. J Pathol 2001; 195: 367-374.

17 Quarck R, Nawrot T, Meyns B, et al. C-reactive protein: a new predictor of adverse outcome in pulmonary arterial hypertension. J Am Coll Cardiol 2009; 53: 1211-1218.

18 Wynants M, Quarck R, Ronisz A, et al. Effects of C-reactive protein on human pulmonary vascular cells in chronic thromboembolic pulmonary hypertension. Eur Respir J 2012; 40: 886-894.

19 Wynants M, Vengethasamy L, Ronisz A, et al. NF- $\kappa B$ pathway is involved in CRP-induced effects on pulmonary arterial endothelial cells in chronic thromboembolic pulmonary hypertension. Am J Physiol Lung Cell Mol Physiol 2013; 305: L934-L942.

20 Lang IM, Pesavento R, Bonderman D, et al. Risk factors and basic mechanisms of chronic thromboembolic pulmonary hypertension: a current understanding. Eur Respir J 2013; 41: 462-468.

21 Waltham M, Burnand KG, Collins M, et al. Vascular endothelial growth factor and basic fibroblast growth factor are found in resolving venous thrombi. J Vasc Surg 2000; 32: 988-996.

22 Waltham M, Burnand KG, Collins M, et al. Vascular endothelial growth factor enhances venous thrombus recanalisation and organisation. Thromb Haemost 2003; 89: 169-176.

23 Alias S, Redwan B, Panzenböck A, et al. Defective angiogenesis delays thrombus resolution: a potential pathogenetic mechanism underlying chronic thromboembolic pulmonary hypertension. Arterioscler Thromb Vasc Biol 2014; 34: 810-819.

24 Thistlethwaite PA, Mo M, Madani MM, et al. Operative classification of thromboembolic disease determines outcome after pulmonary endarterectomy. J Thorac Cardiovasc Surg 2002; 124: 1203-1211.

25 Skoro-Sajer N, Marta G, Gerges C, et al. Surgical specimens, haemodynamics and long-term outcomes after pulmonary endarterectomy. Thorax 2014; 69: 116-122.

26 Blauwet LA, Edwards WD, Tazelaar HD, et al. Surgical pathology of pulmonary thromboendarterectomy: a study of 54 cases from 1990 to 2001. Hum Pathol 2003; 34: 1290-1298.

27 Pepke-Zaba J, Delcroix M, Lang I, et al. Chronic thromboembolic pulmonary hypertension (CTEPH): results from an international prospective registry. Circulation 2011; 124: 1973-1981.

28 Owens GK, Kumar MS, Wamhoff BR. Molecular regulation of vascular smooth muscle cell differentiation in development and disease. Physiol Rev 2004; 84: 767-801.

29 Sobue K, Hayashi K, Nishida W. Molecular mechanism of phenotypic modulation of smooth muscle cells. Horm Res 1998; 50: Suppl. 2, 15-24.

30 Firth AL, Yao W, Ogawa A, et al. Multipotent mesenchymal progenitor cells are present in endarterectomized tissues from patients with chronic thromboembolic pulmonary hypertension. Am J Physiol Cell Physiol 2010; 298: C1217-C1225.

31 Yao W, Firth AL, Sacks RS, et al. Identification of putative endothelial progenitor cells (CD34+CD133+Flk-1+) in endarterectomized tissue of patients with chronic thromboembolic pulmonary hypertension. Am J Physiol Lung Cell Mol Physiol 2009; 296: L870-L878. 
32 Humbert M, Morrell NW, Archer SL, et al. Cellular and molecular pathobiology of pulmonary arterial hypertension. J Am Coll Cardiol 2004; 43: 13S-24S.

33 Hassoun PM, Mouthon L, Barberà JA, et al. Inflammation, growth factors, and pulmonary vascular remodeling. J Am Coll Cardiol 2009; 54: S10-S19.

34 Haoming S, Lemin W, Zhu G, et al. T cell-mediated immune deficiency or compromise in patients with CTEPH. Am J Respir Crit Care Med 2011; 183: 417-418.

35 Frey MK, Alias S, Winter MP, et al. Splenectomy is modifying the vascular remodeling of thrombosis. J Am Heart Assoc 2014; 3: e000772.

36 Kabutomori O, Kanakura Y, Iwatani Y. Movement of toxic granulation neutrophils and C-reactive protein in inflammatory processes. Am J Med 2002; 112: 595-596.

37 Kabutomori O, Iwatani Y, Kanakura Y. Toxic granulation neutrophils and C-reactive protein. Arch Intern Med 2000; 160: 3326-3327.

38 Bisoendial RJ, Birjmohun RS, Akdim F, et al. C-reactive protein elicits white blood cell activation in humans. Am J Med 2009; 122: 582.e1-9.

39 Heuertz RM, Tricomi SM, Ezekiel UR, et al. C-reactive protein inhibits chemotactic peptide-induced p38 mitogen-activated protein kinase activity and human neutrophil movement. J Biol Chem 1999; 274: 17968-17974.

40 Zhong W, Zen Q, Tebo J, et al. Effect of human C-reactive protein on chemokine and chemotactic factor-induced neutrophil chemotaxis and signaling. J Immunol 1998; 161: 2533-2540.

41 Inoue $\mathrm{T}$, Kato $\mathrm{T}$, Uchida $\mathrm{T}$, et al. Local release of $\mathrm{C}$-reactive protein from vulnerable plaque or coronary arterial wall injured by stenting. J Am Coll Cardiol 2005; 46: 239-245.

42 Abe N, Osanai T, Fujiwara T, et al. C-reactive protein-induced upregulation of extracellular matrix metalloproteinase inducer in macrophages: inhibitory effect of fluvastatin. Life Sci 2006; 78: 1021-1028.

43 Johnson C, Sung HJ, Lessner SM, et al. Matrix metalloproteinase-9 is required for adequate angiogenic revascularization of ischemic tissues: potential role in capillary branching. Circ Res 2004; 94: 262-268.

44 Modarai B, Burnand KG, Humphries J, et al. The role of neovascularisation in the resolution of venous thrombus. Thromb Haemost 2005; 93: 801-809.

45 Zabini D, Nagaraj C, Stacher E, et al. Angiostatic factors in the pulmonary endarterectomy material from chronic thromboembolic pulmonary hypertension patients cause endothelial dysfunction. PLoS One 2012; 7: e43793.

46 Pulido T, Adzerikho I, Channick RN, et al. Macitentan and morbidity and mortality in pulmonary arterial hypertension. N Engl J Med 2013; 369: 809-818.

47 Rahnavardi M, Yan TD, Cao C, et al. Pulmonary thromboendarterectomy for chronic thromboembolic pulmonary hypertension: a systematic review. Ann Thorac Cardiovasc Surg 2011; 17: 435-445.

48 Hoey ET, Mirsadraee S, Pepke-Zaba J, et al. Dual-energy CT angiography for assessment of regional pulmonary perfusion in patients with chronic thromboembolic pulmonary hypertension: initial experience. AJR Am $J$ Roentgenol 2011; 196: 524-532.

49 Dorfmüller P, Günther S, Ghigna MR, et al. Microvascular disease in chronic thromboembolic pulmonary hypertension: a role for pulmonary veins and systemic vasculature. Eur Respir J 2014; 44: 1275-1288. 